\title{
Relativistic jets at high energies
}

\author{
Amir Levinson ${ }^{1}$ \\ ${ }^{1}$ School of Physics and Astronomy, Tel Aviv University \\ Tel Aviv 69978, Israel \\ email: levinson@wise.tau.ac.il
}

\begin{abstract}
A recent progress in the study of $\gamma$-ray jets is reviewed, with a focus on some theoretical interpretations of the VHE emission from M87, and possibly other misaligned blazars; the connection between the GeV breaks exhibited by bright LAT blazars and opacity sources in the broad line region; the consequences of the detection of $\mathrm{GeV}$ emission from GRBs to models of magnetic outflows; and the implications of the thermal emission observed is some GRBs to dissipation of the outflow bulk energy.
\end{abstract}

Keywords. Jets, blazars, gamma ray bursts.

\section{Introduction}

Observations by Fermi and the various TeV experiments, and advances in numerical techniques have led to a progress in our understanding of relativistic jets: i) In M87, combined VLBA and TeV data (Acciari et al. 2009) seem to indicate that the TeV emission is produced on horizon scales by either some magnetospheric process, or at the base of the VLBA jet. The observational constraints raise interesting questions about the structure of the BH magnetosphere and the jet formation mechanism, that appear to be relevant also to other TeV AGNs. ii) The LAT spectrum of blazars provide now a better than before probe of opacity sources on sub-parsec scales. The data reveal relationship between source power and spectral features that can be interpreted in terms of BLR properties. iii) Combined Fermi and $\mathrm{TeV}$ observations of VHE blazars may be used to probe intergalactic magnetic fields (e.g., Neronov et al. 2010; Tavecchio et al. 2010). Attempts to derive constrains on the IGMF have been published, and although inconclusive yet, the results demonstrate the potential in pursuing further these efforts. iv) Fermi LAT detections of several GRBs indicate very high Lorentz factors in outflows having opening angles larger than the causality scale. Those detections triggered recent theoretical studies and numerical simulations of magnetic outflows. The conclusion emerging from these studies is that processes beyond ideal MHD are crucial. Hydrodynamical outflows, perhaps driven by neutrino annihilation, may provide an alternative. The nature of the GeV emission in GRBs is yet an open issue. v) In several bursts a prominent, quasi-thermal spectral component has been detected, challenging the "standard model". It opens up the issue of dissipation and emission during the prompt phase. In particular, part of the emission is likely produced behind relativistic radiation mediated shocks.

In what follows, these issues are discussed in some greater detail.

\section{GeV-TeV emission from AGNs}

Over 700 AGNs, most of which are blazars, are listed in the first Fermi LAT catalogue (Abdo, et al. 2010c). Around 3 dozens AGNs have been detected by various TeV experiments at energies above $100 \mathrm{GeV}$, with a spectrum extending up to $10 \mathrm{TeV}$ in some 
cases. At least 50 percents of the TeV sources have GeV counterparts. The LAT spectrum of many bright Fermi blazars exhibits curvature or breaks at GeV energies (Abdo et al. 2010b), conceivably associated with attenuation by radiation emitted from highly ionized clouds in the broad line region (BLR).

Eleven non-blazar AGNs, - 7 FRI radio galaxies and 4 FRII radio sources - exhibit VHE (> $100 \mathrm{MeV}$ ) emission (Abdo, et al. 2010a), and some of them, e.g., M87 (Wagner et al. 2009), Cen A (Aharonian, et al. 2009), are also TeV sources. According to the unified model, those radio galaxies are misaligned blazars, and the question then arises if the VHE photons seen represent side emission from the jet, or have a different origin. In the former case, the high luminosities measured in some of the objects may imply surprisingly small Doppler factors, or extremely high jet power.

It is conceivable that the misaligned blazars exhibit spectral components that are difficult to detect in blazars because they are overwhelmed by the beamed emission from jet. Emission from a starved magnetosphere is one possibility (Levinson 2000). Recent VLBA and TeV observations of M87 (Acciari et al. 2009) strongly motivates reconsideration of this scenario. Below we propose that the variable TeV emission observed in M87 could be a manifestation of the jet formation process.

\subsection{Magnetospheric TeV emission and jet formation in $M 87$}

Combined VLBA and TeV observations of M87 reveal a rapidly varying TeV emission that appears to be associated with the m.a.s VLBA jet. The rapid flaring activity of the $\mathrm{TeV}$ source, with timescales $t=1 t_{d a y}$ day as low as 1-2 days, implies a source size of $d \sim$ $4 r_{s} t_{d a y}$ for a black hole mass $M_{B H}=4 \times 10^{9}$ solar masses. This, and the fact that the TeV emission appears to be correlated with the VLBA jet but not with emission from larger scales (and in particular HST-1), motivates the consideration that the observed TeV rays originate from the black hole magnetosphere. A plausible magnetospheric process discussed in the literature is curvature and/or IC emission by particles, either hadrons or leptons, accelerating in a vacuum gap of a starved magnetosphere. An alternative explanation is emission from small regions located at larger radii, $r \sim 100 r_{g}$, as, e.g., in the misaligned minijets model of Gainnios et al. (2010).

The presence of the VLBA jet implies that a force-free (or ideal MHD) flow is established on scales $<100 r_{g}$, so that the magnetosphere is anticipated to be screened in the sense that the invariant $\mathbf{E} \cdot \mathbf{B}$ nearly vanishes everywhere. However, as in pulsar theory, there must be a plasma source that compensates for the loss of particles which escape the system (both, to infinity and across the horizon) along the open magnetic field lines in the polar region. The nature of this plasma source is poorly understood at present.

The injection of charges into the magnetosphere may be associated with the accretion process. Direct feeding seems unlikely, as charged particles would have to cross magnetic field lines on timescale shorter than the accretion time in order to reach the polar outflow. Free neutrons, if exist, decay over length scale much smaller than the gravitational radius of the black hole. Annihilation of $\mathrm{MeV}$ photons produced in the radiative inefficient flow, may provide the required charge density, depending on accretion rate and other details.

A possible plasma source is cascade formation in starved magnetospheric regions. The size of the gap then depends on the conditions in the magnetosphere and the pair production opacity. As shown elsewhere (Levinson 2000), vacuum breakdown by back-reaction is unlikely, as it requires magnetic field strength in excess of a few times $10^{5} \mathrm{G}$, higher than the equipartition value for Eddington accretion. Pair production via absorption of $\mathrm{TeV}$ photons by the ambient radiation field is more likely. However, the spectrum of the VHE photons observed by HESS extends up to $10 \mathrm{TeV}$, and the assumption that these photons originate from the magnetosphere (or even the VLBA jet) implies that the 


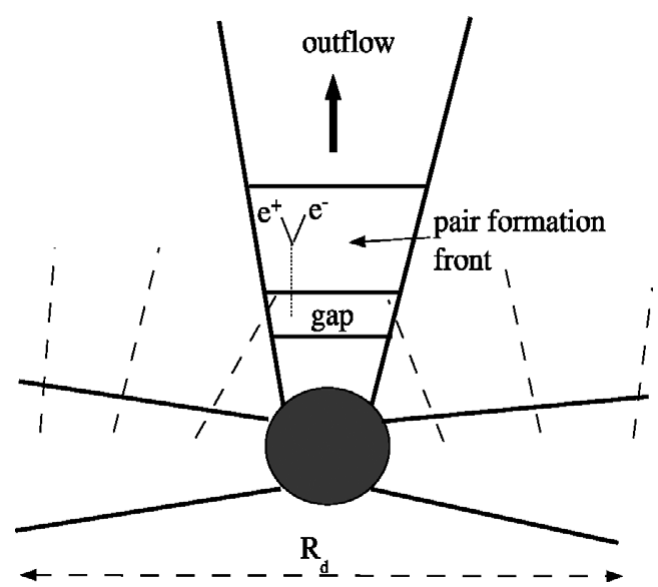

Figure 1. Schematic representation of the magnetosphere structure: A vacuum gap of height $h<r_{g}$ accelerates particles (electrons or positrons) to high Lorentz factors. The gap is exposed to soft radiation emitted from a source of size $R_{d}>>r_{g}$. Curvature emission and inverse Compton scattering of ambient radiation produce VHE photons with a spectrum extending up to $10^{4}$ $\mathrm{TeV}$. Photons having energies below a few TeV can escape freely to infinity. Interactions of IC photons having energies well above $10 \mathrm{TeV}$ with the ambient radiation initiate pair cascades just above the gap, leading to a large multiplicity. A force free outflow is established just above the pair formation front, and appears as the VLBA jet. Intermittencies of the cascade process give rise to the observed variability of the $\mathrm{TeV}$ emission, and the resultant force-free outflow, as indicated by the morphological changes of the VLBA jet.

pair production opacity at these energies must not exceed unity. This raises the question whether pair cascades in the magnetosphere can at all account for the multiplicity required to establish a force-free flow.

In an upcoming paper (Levinson \& Rieger, 2010, in preparation) it is shown that inverse Compton scattering (IC) of ambient photons by electrons accelerated in the gap can lead to a large multiplicity, in excess of $10^{3}$, while still allowing photons at energies of up to a few $\mathrm{TeV}$ to freely escape the system. The seed electrons are generated by the $\mathrm{MeV}$ emission from the RIAF. This requires that the ambient radiation source contributing the opacity will have a characteristic size $R_{d} \sim 10^{2} r_{g}$. Preliminary results indicate that the electromagnetic cascade is initiated by IC photons having much higher energies, $\sim 10^{3}$ $\mathrm{TeV}$, for which the $\gamma \gamma$ optical depth is much larger. It is found that the gap width is not much smaller than $0.1 r_{g}$. The luminosity of the VHE photons produced in the gap, roughly a fraction $\left(h / r_{g}\right)^{2}$ of the maximum BZ power, can easily account for the $\mathrm{TeV}$ luminosity observed by HESS and Fermi. Any intermittencies of the cascade formation process would naturally lead to variability of both, the magnetospheric $\mathrm{TeV}$ emission and the resultant force-free flow, as observed. A schematic illustration of the model is presented in figure 1.

This model may be applicable also to some of the other misaligned VHE blazars.

\subsection{GeV breaks and opacity sources in blazars}

The nature of opacity sources in blazars is an issue of considerable interest, particularly in regards to the location of the VHE emission zones. Much efforts have been devoted over the years to identify different opacity sources, and detailed calculations of $\gamma \gamma$ attenuation by these sources are presented in the literature. Recent Fermi observations have led to reconsideration of the effect of the broad line region on the $\mathrm{GeV}$ spectrum of blazars. 
Strong departure from a single power law appears to be a common feature in FSRQs (Abdo et al. 2010b). The break energies, typically lying in the energy range 2-10 GeV, are too low to be due to absorption by Ly $\alpha$ radiation. Recently, it has been pointed out (Putanen \& Stern 2010) that the LAT spectrum of FSRQs can be well reproduced by a double absorber model. Detailed numerical calculations of the BLR spectrum for different ionization parameters indicates that the observed $\mathrm{GeV}$ breaks can be accounted for by $\gamma$-ray absorption through pair production on He II and H I recombination continua. The exact location of the break energy depends on the ionization parameter, as described in Putanen \& Stern (2010). The fact that GeV breaks are seen mainly in the brightest blazars is naturally explaned by the scaling of the BLR size with luminosity, $R_{B L R} \propto$ $L^{1 / 2}$, obtained from reverberation mapping techniques (Kaspi et al. 2007), that implies $\tau_{\gamma \gamma} \propto L^{1 / 2}$.

\section{VHE emission and dissipation in GRBs}

Over a dozen GRBs, both long and short, have been detected thus far by LAT onboard Fermi. The origin of the GeV emission is yet an open issue. The flux level of the LAT emission and its long lasting light curve, suggest that this component is produced during the afterglow phase, although other explanations have been offered. For sources that show sub-second variations of the GeV flux, e.g., 080916C, opacity arguments yield high Lorentz factors, $\Gamma \sim 10^{3}$. While various factors render those estimates uncertain (e.g., Yuan-Chuan et al. 2010), it seems difficult to avoid the conclusion that the outflow reaches high Lorentz factors. Such high Lorentz factors posses a great challenge to jet models, as discussed in the following.

Another interesting result is that in some GeV bursts, e.g., GRB 090902B (Pe'er et al. 2010), a prominent quasi-thermal spectral component has been detected, whereas in others, e.g., 080916C, a fit by a Band spectrum is claimed to be satisfactory, and there appears to be no indication for thermal emission. This, presumably, reflects the conditions in the dissipation region. Below it is argued that in bursts that exhibit a thermal component a substantial fraction of the outflow bulk energy dissipates below the photosphere, in relativistic radiation mediated shocks, whereas in other bursts most of the energy dissipates above the photosphere, behind collisionless shocks.

\subsection{Formation and acceleration of magnetic jets}

Extraction of rotational energy from a rapidly spinning black hole by magnetic fields is a widely discussed jet production mechanism (e.g., Levinson \& Eichler 1993; Lyutikov \& Blandford 2003; Giannios \& Spruit 2005). Up to 30 percents of the black hole mass can be extracted from a maximally rotating hole, which is more than sufficient to account for the energetics of essentially all GRBs. The rate at which the energy is extracted depends on the strength of the magnetic field in the vicinity of the horizon. Recent numerical simulations confirm that a Blandford-Znajek process does operate in Kerr spacetime, and that for reasonable assumptions about the progenitor star, sufficiently large power can be extracted in the form of an outflow. Moreover, a very low baryonic content is anticipated along horizon threading field lines, allowing, in principle, high asymptotic Lorentz factors of the polar outflow. Pressure support by the external medium may give rise to collimation of the central outflow (Bromberg \& Levinson 2007; Lyubarsky 2009). Magnetic outflows have been considered also in the context of the unexpected paucity of optical flashes seen in GRBs (e.g., Zhang \& Kobayashi 2005; Mimica et al. 2009), and it has been shown that even moderate magnetization $(\sigma \sim 0.3)$ would strongly suppress the reverse shock. The question remains as to how the internal shocks that produce the 
prompt emission form under these conditions. Alternative explanations for the paucity of optical flashes, e.g., an early onset of a R-T instability (Levinson, 2009, 2010a), have been offered recently.

An key issue in the theory of magnetically dominated outflows is magnetic energy dissipation. The process by which magnetic energy is converted into kinetic energy has not been identified yet. Stationary magnetic outflows allow, in general, only partial conversion of magnetic-to-kinetic energy. For a split monopole field acceleration ceases at an asymptotic Lorentz factor $\Gamma_{\infty} \sim \sigma_{0}^{1 / 3}$, and magnetization $\sigma_{\infty} \sim \sigma_{0}^{2 / 3}$, where $\sigma_{0}$ is the initial magnetization of the expanding shell. A better conversion can be achieved if the outflow is collimated into a small opening angle, $\theta \simeq \Gamma^{-1}$ (Komissarov et al. 2009). Such small opening angles are problematic, particularly for the GeV bursts, for which $\Gamma_{\infty} \sim 500-1000$ is inferred. Corking from a star that, within the framework of the collapsar model, may be relevant to long bursts can alleviate the latter condition (Tchekhovskoy et al., 2010; Komissarov et al. 2010). However, even then $\sigma_{\infty}$ cannot be much smaller than unity (Lyubarski 2010). Furthermore, there is evidence for extreme Lorentz factors also in short bursts, where strong deconfinement is not anticipated.

Recently it has been shown (Granot et al., 2010; hereafter GKS10; Lyutikov 2010a,b) that time-dependent effects may play a crucial role in the acceleration of a magnetized flow. Unlike a stationary flow, for which acceleration ceases at $\Gamma_{\infty} \sim \sigma_{0}^{1 / 3}$, an impulsive spherical shell expelled by a central source continues accelerating, even after loosing causal contact with the engine, until reaching nearly complete conversion of magnetic energy into bulk kinetic energy. The terminal Lorentz factor of a shell expanding in vacuum is $\Gamma_{\infty} \simeq \sigma_{0}$. During the acceleration phase the major fraction of the shell energy is contained in a layer of width $2 r_{0}$, where $r_{0}$ is the initial shell's width, bounded between the front of a rarefaction wave reflected from the central source and the head of the shell. The average Lorentz factor of the shell, roughly equals the Lorentz factor of the fluid at the rarefaction front, evolves as $\left\langle\Gamma>\propto t^{1 / 3}\right.$. Once the shell enters the coasting phase its width starts growing and its magnetization continues to drop, until nearly full conversion is accomplished.

However, for sufficiently high values of the initial magnetization $\sigma_{0}$ the evolution of the system is significantly altered by the ambient medium well before the shell reaches its coasting phase (Levinson 2010b). The maximum Lorentz factor of the shell is limited to values well below $\sigma_{0}$. To be concrete, for a shell of initial energy $E=10^{52} E_{52}$ erg and size $r_{0}=10^{12} T_{30} \mathrm{~cm}$ expelled into a medium having a uniform density $n_{i}$ the Lorentz factor is limited to $\Gamma_{\max } \simeq 180\left(E_{52} / T_{30}^{3} n_{i}\right)^{1 / 8}$ in the high sigma limit. The reverse shock and any internal shocks that might form if the source is fluctuating are shown to be very weak. The restriction on the Lorentz factor is more severe for shells propagating in a stellar wind. Intermittent ejection of multiple, thin shells does not seem to help, as even in vacuum unlikely small duty cycle is required in order for shells to collide after reaching the coasting phase. Such episodes are expected to produce a smooth, relatively fast rising slowly decaying (power law) light curve, even in a multi-shell scenario. Events like GRB080916C and GRB090510 (Abdo et al. 2009a,b) are not easily accounted for by the impulsive high-sigma shell model.

The main conclusion from those recent studies of magnetic outflows is that processes beyond ideal MHD, as might occur in e.g., a striped wind model, are required.

\subsection{Thermal emission and relativistic radiation mediated shocks}

Shocks that form by overtaking collisions can dissipate energy at radii $r_{d}>\Gamma^{2} c \delta t$, where $\Gamma$ is the Lorentz factor of the slow shell and $\delta t \geqslant r_{s} / c$ is time interval between ejections of the two consecutive shells. In blazars and microquasars with $\Gamma \sim 1-50$ dissipation by 


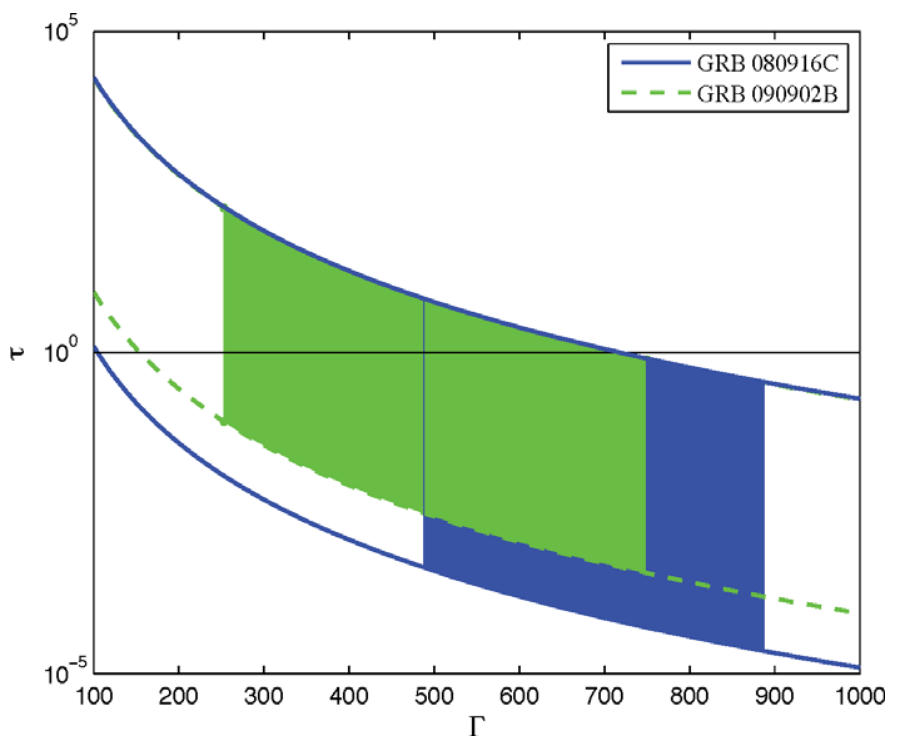

Figure 2. Optical depth at the shells' collsion radius as a function of Lorentz factor, for different values of $\delta t$, the time interval between ejections of the two consecutive shells. The upper curves correspond to the dynamical time of a 3 solar mass black hole, $\delta t \simeq 10^{-4} \mathrm{~s}$. The lower curves are limits from observations, with $\delta t=0.5 \mathrm{~s}$ for GRB080916C, and $\delta t=0.05 \mathrm{~s}$ for GRB090902B. The range of $\Gamma$ in each source represents various estimates taken from the literature (Bromberg et al. 2010).

internal shocks is expected close to the $\mathrm{BH}$, consistent with (but not necessarily implied by) the short durations of strong flares observed in these objects, particularly in $\mathrm{TeV}$ blazars.

In GRBs $r_{d}>10^{5} r_{s}$ or so for the Lorentz factors envisaged. A rough condition for internal shocks to form above the photosphere is $\Gamma>200 L_{52}^{1 / 5} \delta t_{-3}^{-1 / 5}$, where $L_{52}$ is the burst luminosity in units of $10^{52} \mathrm{erg} / \mathrm{s}$, and $\delta t_{-3}=\delta t /(1 \mathrm{~ms})$. Results of more exact calculations (Bromberg et al. 2010) are exhibited in figure 2, where the Thompson optical depth at the radius of shock formation, $\tau$, is plotted agains the flow Lorentz factor $\Gamma$. The two colored areas correspond to a range of $\delta t$ and $\Gamma$ for GRB 080916C (blue) and GRB 090902B (green), as explained in the figure caption.

As shown, in case of GRB 080916C internal shocks are expected to form above the photosphere for shells accelerated to $\Gamma>700$. Such shocks are probably collisionless, and can Fermi accelerate particles to nonthermal energies. Slower shells ejected over time intervals of the order of the dynamical time will collide below the photosphere, at a moderate optical depth $\tau<100$. The parameter space explored in Figure 2 indicates that in this burst the majority of the explosion energy dissipates above the photosphere, behind collisionless shocks, so that a thermal component may be very weak or absent.

In GRB 090902B, on the other hand, the major fraction of the energy is likely to dissipate below the photosphere, albeit in a region of moderate optical depth, $\tau<300$, as seen in Figure 2. Only sufficiently slow shells that have large separations, such $r_{s} / \delta t$ is much larger than the anticipated duty cycle of the engine, will collide above the photosphere to form collisionless shocks. The shocks that form below the photosphere, where the Thomson depth exceeds unity, are mediated by Compton scattering (Bromberg \& Levinson 2008; Katz et al. 2010). Under conditions anticipated in GRBs, such relativistic radiation mediated shocks (RRMS) convect enough radiation upstream to render photon 
production in the shock transition negligible (Bromberg, et al., 2010), unlike the case of shock breakout in supernovae and hypernovae (Katz et al. 2010). Bulk Comptonization then produces a relatively low thermal peak, followed by a broad, nonthermal component in the immediate downstream that extends up to the KN limit in the shock frame (or up to $\sim \Gamma m_{e} c^{2}$ in the observer frame), and perhaps even beyond (Budnik et al. 2010), depending on details. The radiation produced downstream is trapped for times much longer than the shock crossing time, and diffuses out after the shock breaks out of the photosphere and becomes collisionless. At what depth downstream equilibrium is established is yet an open issue. Since the enthalpy downstream is dominated by radiation, a full equilibrium is not expected for the moderate optical depths found above. We contend that a thermal component plus a hard tail, as seen in GRB 090902B, can be emitted from the downstream of a RRMS. A full treatment requires detailed transfer calculations, or Monte-Carlo simulations.

\section{Acknowledgement}

I thank Omer Bromberg and Yuri Lyubarsky for enlightening discussions. This work was supported by an ISF grant for the Israeli Center for High Energy Astrophysics.

\section{References}

Abdo, A. A., et al. 2009a, Science, 323, 1688

Abdo, A. A., et al. 2009b, arXiv0908.1832

Abdo A. A. et al., 2010a ApJ, 720, 912

Abdo A. A. et al., 2010b $A p J, 710,1271$

Abdo A. A. et al., 2010c ApJS, 188, 405

Acciari, V. A. et al. 2009, Science, 325, 444

Aharonian, F., et al. (H.E.S.S. Collaboration) 2009, ApJ, 695, L40

Bromberg, O. \& Levinson, A. 2007, ApJ, 671, 678

Bromberg, O., Mikolitzky, Z., \& Levinson, A. 2010, (to be submitted)

Budnik, R. et al. 2010, arXiv:1005.0141

Giannios D. \& Spruit H., 2005, A\& $A, 430,1$

Giannios, D., Uzdensky, D. A., \& Begelman, M. C., 2010, MNRAS, 402, 1649

Granot, J., Komissarov S., \& Spitkovsy, A., 2010, arXiv:1004.0959

Kaspi, S. et al. 2007, ApJ, 659, 997

Katz, B. Budnik, R. \& Waxman, E. 2010, ApJ, 716, 781

Levinson, A. 2000, Phys. Rev. Lett, 85, 912

Levinson, A. 2009, ApJ, 705, 213

Levinson, A. 2010, GApFD, 104, 84

Levinson, A. 2010, ApJ, 720, 1490

Levinson A. \& Eichler D., 1993, ApJ, 418, 386

Levinson, A. \& Bromberg, O. Phys. Rev. Lett., 100 (2008) 131101

Lyubarsky, y. 2009, ApJ, 698, 1570

Lyubarsky, y. 2010, MNRAS, 402, 353

Lyutikov M. \& Blandford R. D. 2003, arXiv:astro-ph/0312347

Lyutikov M. 2010a, arXiv:1004.2428

Lyutikov M. 2010b,arXiv:1004.2429

Mimica, P., Giannios, D., \& Aloy, M. A., 2009, A\&A, 494, 879

Neronov, A. et al. 2010, ApJ, 719, L130

Peer, A. et al. 2010, arXiv:1007.2228

Poutanen, J. \& Stern, B. 2010, ApJ, 717, L118

Tavecchio, F. et al. 2010, MNRAS, 406, L70

Tchekhovskoy, A., Narayan, R., \& McKinney, J. C. 2010, New Astron. 
Wagner, R. M. et al. 2009, Proc. ICRC, arXiv:090\%.1465

Yuan-Chuan, Z., Yi-Zhong, F., \& Piran, T. 2010 arXiv:1008.2253

Zhang, B. \& Kobayashi, S. 2005, ApJ, 628, 315

\section{Discussion}

Romero: Can you please elaborate a bit about the possible implications of "dark" blazars for UHECRs?

LEVINSON: Confinement of an UHECR requires certain magnetic field. This gives a lower limit on the Poynting flux, and, hence, on the jet power. The implied jet power is very high, and if the radiative efficiency of the jet is not small, the source must be bright. There is no evidence for such bright sources within the GZK sphere, so the only way around it is to assume "dark" sources.

KIRK: You stated that the acceleration of a magnetic shell is inhibited by an ambient medium. Is it possible that in a GRB the first shell evacuates a channel and subsequent shells are accelerated to high Lorentz factors in a very low density environment?

LEVINSON: It is in principle possible if the duty cycle is extremely small, which I find unlikely. The point is that even in vacuum, for reasonable separation between shells they will collide while still highly magnetized, because the fron moves faster than the inner regions.

PE'ER: How well is the Lorentz factor in M87 constrained, and can Lorentz aberration help resolve the "small scale" problem of the $\mathrm{TeV}$ emission?

Levinson: Not well, SL motions imply $\Gamma>6$ on certain scales. However, the viewing angle is not small, $\sim 20^{\circ}-30^{\circ}$, so the Doppler factor is always small. 